\title{
El Comportamiento Etnocentrista De Los \\ Consumidores Jóvenes En Saltillo, Coahuila
}

\author{
Dra. Adriana Méndez Wong \\ Mtra. Elizabeth Ana Aguilar Garcés \\ Mtra. Juana Alicia Villarreal Cavazos \\ Frida María Martínez Ramírez. \\ Universidad Autónoma de Coahuila, Mexico
}

Doi:10.19044/esj.2018.v14n31p122 URL:http://dx.doi.org/10.19044/esj.2018.v14n31p122

\begin{abstract}
This paper focuses on identifying the young people that show predisposition when consuming foreign products. A Cestscale instrument was applied (Consumer Ethnocentric Tendencies Scale) and developed by Tetence A. Shimp and Sharma S. (1987), and was quoted by Schiffman and Lazar Kanuk (2010). The questions were categorical and the answers were pointed on a Likert scale from 1 to7. The study is descriptive and exploratory with a transversal no experimental measurement. 103 valid polls were obtained with a rate of $95 \%$ validating the 17 items instrument with a Cronbach Alpha of 0870. The study results show that the interviewed population considered themselves as an ethnocentric buyer. This is because it evaluated the answers to the variant to be high. Also, it statistically confirms that there are no meaningful differences between men and women in regards to their ethnocentric characteristics.
\end{abstract}

Keywords: Shopping behavior, Etnocentrist consumer, CETSCALE

\section{Resumen}

El presente trabajo pretende identificar a los jóvenes que muestran predisposición a consumir productos de origen extranjero. Para tal fin se aplica un instrumento utilizando la escala CETSCALE (Consumer Ethnocentric Tendencies Scale) desarrollada por Terence A. Shimp y Sharma S. (1987) y citada por Schiffman y Lazar Kanuk (2010). Las preguntas fueron categóricas y las respuestas se indicaron en escala de Likert de 1 a 7 . La investigación es de carácter descriptivo y exploratorio con medición transversal no experimental, investigación en la cualse obtuvieron 103 encuestas válidas con una tasa de respuesta del 95\%, validando el instrumento de 17 ítems con un Alfa de Cronbach de 0.870. Los resultados del estudio reflejan que la población 
entrevistada si se considera comprador etnocéntrico, pues evalúa alto las respuestas a las variables que así lo sugieren. Además, se confirma estadísticamente que no hay diferencias significativas entre hombres y mujeres con respecto a sus características etnocéntricas.

Palabras Claves: Comportamiento de Compra, Consumidor Etnocentrista, CETSCALE

\section{Introducción}

La influencia de la globalización en el aumento del comercio mundial ha generado que los consumidores se enfrenten a un mayor número de productos provenientes de países con diferencias significativas a los que ofertan los mercados locales. Esto resulta evidentemente en un proceso más complejo en la toma de decisiones de los clientes.

Con el fin de identificar a los consumidores que prefieren productos hechos en el extranjero, los investigadores han desarrollado y probado empíricamente una escala de Etnocentrismo del Consumidor, conocida como CETSCALE (Consumer Ethnocentric Tendencies Scale) propuesta por Shimp y Sharma (1987). Según Schiffman y Lazar Kanuk (2010) dicha escala ha resultado exitosa en la identificación de los consumidores que muestran predisposición a aceptar (o a rechazar) bienes de manufactura extranjera, y ha demostrado ser una medida confiable tanto en Estados Unidos como en otras naciones. Según dicha escala, los consumidores que son altamente etnocéntricos suelen considerar que es incorrecto o erróneo comprar productos hechos en el extranjero a causa del impacto financiero resultante en la economía nacional; en tanto que los consumidores no etnocéntricos tienden a valorar — de una forma mucho más objetiva - los productos de fabricación extranjera considerando sus características extrínsecas (por ejemplo, ¿qué tan buenos son?). De igual manera, una investigación posterior al 11 de septiembre en Estados Unidos que buscaba evaluar las actitudes de la Generación Y (quienes nacieron entre 1977 y 1994) hacia las marcas extranjeras y nacionales, reveló que había pocas señales de etnocentrismo en este grupo, mientras que en otro estudio se encontró que para los consumidores en general, el etnocentrismo aumentó como resultado de los ataques terroristas de 2001 y por los desastres naturales.

\section{Revisión de literatura}

El etnocentrismo en el consumo es la preferencia del consumidor por productos nacionales generando lealtad hacia su país y mostrando rechazo hacia otros países (Chung, Boyer, \& Han, 2009). Es la creencia en la superioridad de las prácticas y los productos del propio país. Los consumidores etnocéntricos sentirán que es incorrecto comprar productos de otros países, en 
especial debido al efecto negativo que esto puede tener en la economía doméstica (Solomon, 2008).

La literatura a nivel internacional nos brinda antecedentes de investigaciones donde los clientes evalúan los productos considerando los países donde fueron producidos, y que en general, los clientes prefieren las marcas domésticas o locales. Con el fin de comprender el comportamiento patriótico de consumo, se identifican dos líneas o posturas teóricas para la investigación: estudios referidos al "efecto país de origen" y trabajos enfocados en el etnocentrismo en el consumo. (Shimp y Sharma (1987) y Shankarmahesh (2006) citados por Schnettler, Cid, Miranda, Sepúlveda \& Denegri (2010)).

Luego de una intensa investigación que permitió la creación del cuestionario CETSCALE como un instrumento para medir estas actitudes en los consumidores, los investigadores concluyeron que, desde la perspectiva etnocéntrica, la compra de productos importados resulta negativa, pues en la mente de los consumidores este tipo de compras se asocia a un atentado contra la economía doméstica, causa pérdida de empleos y es una conducta antipatriótica; es decir, para consumidores con alto nivel de etnocentrismo los productos provenientes de otros países (exogrupos) son objeto de desprecio (Muñoz-Penagos \& Velandia-Morales, 2014).

Según Schiffman y Lazar Kanuk (2010), la evidencia disponible sugiere que el etnocentrismo varía según el país y el producto. Los consumidores mexicanos, por ejemplo, son más etnocéntricos que los franceses y los estadounidenses, y los consumidores malasios, aún cuando prefieren comprar trusas, camisas, ropa interior y cinturones de manufactura local, también buscan adquirir gafas para sol y relojes importados.Otros hallazgos sugieren que algunos consumidores estadounidenses de mayor edad, como recuerdo de la Segunda Guerra Mundial, todavía se rehúsan a comprar productos alemanes y/o japoneses; en tanto que algunos consumidores alemanes y japoneses pueden sentir lo mismo acerca de los bienes estadounidenses. Es más, para algunos consumidores, el país de ensamble y/o el país de diseño también influyen su decisión de comprar o de abstenerse de comprar un artículo específico.

Con razón o sin ella, la política exterior de Estados Unidos tiene gran influencia en la forma en que los consumidores perciben las marcas estadounidenses. Una encuesta de 2004 descubrió que 66 por ciento de los consumidores encuestados en Alemania afirmaronque eran menos propensos a comprar productos estadounidenses debido a que se oponen a la guerra de Estados Unidos contra Irak (Solomon, 2008).

Schnettler, Cid, Miranda, Sepúlveda y Denegri (2010) llevan a cabo un estudio donde evalúan la preferencia en la compra de arroz de distinto país de origen, calidad y precio en consumidores de supermercados de Temuco, y 
Los Angeles, Chile, para también identificar las tipologías de consumidores. Con una muestra de 800 consumidores y mediante un análisis conjunto, determinaron que el país de origen $(55.4 \%)$ fue más importante que la calidad $(22.6 \%)$ y el precio $(22.0 \%)$, con diferencias significativas entre ciudades en la importancia del origen y del precio.

Con base en estos antecedentes, la finalidad del estudio es identificar a los jóvenes que presentan rasgos de comportamiento etnocentrista en la ciudad de Saltillo, Coahuila.

\section{Metodología}

Se aplica un cuestionario desde la perspectiva conceptual de la escala CETSCALE (Consumer Ethnocentric Tendencies Scale) propuesta por Shimp y Sharma (1987).

Muestra. La investigación utilizó una muestra de 103 participantes jóvenes residentes en la ciudad de Saltillo, Coahuila.

METODOLOGÍA (Hernández, Fernández, \& Baptista, 2014)

Tabla 1. Estadísticos de fiabilidad

\begin{tabular}{|l|l|}
\hline $\begin{array}{c}\text { Alfa de } \\
\text { Cronbach }\end{array}$ & $\begin{array}{c}\text { No de } \\
\text { elementos }\end{array}$ \\
\hline .870 & \\
\hline & \\
\hline
\end{tabular}

Para el presente estudio se utiliza la escala CETSCALE, la cual está compuesta de diecisiete ítems y evalúa específicamente tendencias etnocéntricas para investigaciones de mercadeo. Esta utiliza una escala de 7 puntos en formato tipo Likert (totalmente de acuerdo, 7; totalmente en desacuerdo, 1).

\section{Items:}

1 Los mexicanos siempre deberían comprar productos nacionales en vez de importados.

2 Sólo aquellos productos que no estén disponibles en México deberían importarse.

3 Compre productos hechos en México. Mantenga a nuestro país funcionando.

4 Los productos mexicanos están primero, al final y antes que todos.

5 Comprar productos extranjeros es un acto "antimexicano".

6 No es correcto comprar productos extranjeros porque eso origina que algunos mexicanos se queden sin trabajo.

7 Un mexicano verdadero siempre debería comprar productos hechos en México.

8 Deberíamos comprar productos hechos en México en vez de permitir que otros países se enriquezcan a costa nuestra.

9 Siempre es mejor comprar productos mexicanos.

10 Debería haber muy poco comercio o compra de productos de otros países, a menos que se trate de una necesidad absoluta.

11 Los mexicanos no deberían comprar productos extranjeros porque eso daña los negocios mexicanos y causa desempleo. 
12 Debería ponerse límite a todas las importaciones

13 Quizás a la larga me cueste más dinero, pero prefiero ofrecer mi apoyo a los productos mexicanos.

14 No debería permitirse a los extranjeros que introduzcan sus productos en nuestros mercados.

15 Se tiene que gravar significativamente a los productos extranjeros para reducir su ingreso a México.

16 Deberíamos comprar de los otros países solo aquellos productos que no podamos obtener en nuestro propio país.

17 Los consumidores mexicanos que adquieran productos hechos en otros países son responsables por dejar sin empleo a sus conciudadanos.

Escala adaptada CETSCALE (Consumer Ethnocentric Tendencies Scale) propuesta por Shimp y Sharma (1987) citado por Schiffman y Lazar (2010).

\section{Resultados}

Tabla 2. Situación media de los jóvenes encuestados respecto a su comportamiento etnocentrista ${ }^{1}$

\begin{tabular}{|l|c|}
\hline \multicolumn{1}{|c}{ Variables } & Medias \\
\hline Los mexicanos siempre deberían comprar productos nacionales en vez de & 4.81 \\
importados.. & 5.10 \\
Sólo aquellos productos que no estén disponibles en México deberían importarse. & 5.83 \\
Compre productos hechos en México. Mantenga a nuestro país funcionando. & 4.55 \\
Los productos mexicanos están primero, al final y antes que todos & 2.77 \\
Comprar productos extranjeros es un acto "antimexicano". & 3.71 \\
No es correcto comprar productos extranjeros porque eso origina que algunos \\
mexicanos se queden sin trabajo. & 3.83 \\
Un mexicano verdadero siempre debería comprar productos hechos en México. \\
$\begin{array}{l}\text { Deberíamos comprar productos hechos en México en vez de permitir que otros } \\
\text { países se enriquezcan a costa nuestra. }\end{array}$ & 4.52 \\
Siempre es mejor comprar productos mexicanos & 4.35 \\
Debería haber muy poco comercio o compra de productos de otros países, a menos & 3.97 \\
que se trate de una necesidad absoluta. & \\
Los mexicanos no deberían comprar productos extranjeros porque eso daña los & 4.13 \\
negocios mexicanos y causa desempleo. & 4.26 \\
Debería ponerse límite a todas las importaciones & 4.68 \\
Quizás a la larga me cueste más dinero, pero prefiere ofrecer mi apoyo a los \\
productos mexicanos.
\end{tabular}

${ }^{1}$ En una escala de totalmente de acuerdo, 7; totalmente en desacuerdo, 1.

Fuente: Elaboración propia 
La Tabla 2 muestra que las tres principales variables donde los jóvenes se encuentran de acuerdo son en orden de importancia: Ccompre productos hechos en México, Mantenga a nuestro país funcionando, con una media de 5.83 en una escala de $1=$ total desacuerdo a $7=$ total acuerdo como límites, seguida de la variable Sólo aquellos productos que no estén disponibles en México deberían importarse, con una media de $5.10 \mathrm{y}$, finalmente, Deberíamos comprar de los otros países solo aquellos productos que no podamos obtener en nuestro propio país, con una media de 4.87 .

Tabla 3 Prueba de hipótesis. Test Prueba U de Mann-Whitney de muestras independientes considerando la variable Género.

\begin{tabular}{|c|c|c|}
\hline Hipótesis Nula & Medias & Sig. \\
\hline $\begin{array}{l}\text { Los mexicanos siempre deberían comprar productos nacionales en vez de } \\
\text { importados,es independientemente del género. }\end{array}$ & 4.81 & .139 \\
\hline $\begin{array}{l}\text { Sólo aquellos productos que no estén disponibles en México deberían importarse; } \\
\text { es independiente del género. }\end{array}$ & 5.10 & .085 \\
\hline $\begin{array}{l}\text { Compre productos hechos en México. Mantenga a nuestro país funcionando; es } \\
\text { independiente del género. }\end{array}$ & 5.83 & .737 \\
\hline $\begin{array}{l}\text { Los productos mexicanos están primero, al final y antes que todos es independiente } \\
\text { del género. }\end{array}$ & 4.55 & .729 \\
\hline $\begin{array}{l}\text { Comprar productos extranjeros es un acto "antimexicano" independiente del } \\
\text { género. }\end{array}$ & 2.77 & .563 \\
\hline $\begin{array}{l}\text { No es correcto comprar productos extranjeros porque eso origina que algunos } \\
\text { mexicanos se queden sin trabajo, es independiente del género. }\end{array}$ & 3.71 & .945 \\
\hline $\begin{array}{l}\text { Un mexicano verdadero siempre debería comprar productos hechos en México; es } \\
\text { independiente del género. }\end{array}$ & 3.83 & .848 \\
\hline $\begin{array}{l}\text { Deberíamos comprar productos hechos en México en vez de permitir que otros } \\
\text { países se enriquezcan a costa nuestra es independiente del género. }\end{array}$ & 4.52 & .444 \\
\hline Siempre es mejor comprar productos mexicanos ; es independiente del género. & 4.35 & .274 \\
\hline $\begin{array}{l}\text { Debería haber muy poco comercio o compra de productos de otros países, a menos } \\
\text { que se trate de una necesidad absoluta; es independiente del género. }\end{array}$ & 3.97 & .288 \\
\hline $\begin{array}{l}\text { Los mexicanos no deberían comprar productos extranjeros porque eso daña los } \\
\text { negocios mexicanos y causa desempleo ;es independiente del género. }\end{array}$ & 4.13 & .295 \\
\hline Debería ponerse límite a todas las importaciones; es independiente del género & 4.26 & .661 \\
\hline $\begin{array}{l}\text { Quizás a la larga me cueste más dinero, pero prefiere ofrecer mi apoyo a los } \\
\text { productos mexicanos; es independiente del género. }\end{array}$ & 4.68 & .444 \\
\hline $\begin{array}{l}\text { No debería permitirse a los extranjeros que introduzcan sus productos en nuestros } \\
\text { mercados; es independiente del género. }\end{array}$ & 3.00 & .532 \\
\hline $\begin{array}{l}\text { Se tiene que gravar significativamente a los productos extranjeros para reducir su } \\
\text { ingreso a México;es independiente del género. }\end{array}$ & 3.97 & .835 \\
\hline $\begin{array}{l}\text { Deberíamos comprar de los otros países sólo aquellos productos que no podamos } \\
\text { obtener en nuestro propio país; es independiente del género. }\end{array}$ & 4.87 & .532 \\
\hline $\begin{array}{l}\text { Los consumidores mexicanos que adquieran productos hechos en otros países son } \\
\text { responsables por dejar sin empleo a sus conciudadanos; es independiente del } \\
\text { género. }\end{array}$ & 2.97 & .139 \\
\hline
\end{tabular}

${ }^{1}$ En una escala de $1=$ total desacuerdo a $7=$ total acuerdo Se muestran las significancias asintóticas. El nivel de significancia es .05 Fuente: Elaboración propia

Considerando la Tabla 3 se observa que no hay variables en las que exista una diferencia significativa. Por lotanto, no se rechaza las $\mathrm{H} 0$, y se 
puede afirmar con un $95 \%$ de confianza que no hay diferencias significativas entre hombres y mujeres con respecto a sus características etnocéntricas.

Tabla 4. Tabla de contingencia Género y Compre productos hechos en México. ${ }^{1}$

\begin{tabular}{|c|c|c|c|c|c|c|c|c|}
\hline \multirow[b]{2}{*}{ Sexo } & \multicolumn{7}{|c|}{$\begin{array}{l}\text { Compre productos hechos en México. Mantenga a } \\
\text { nuestro país funcionando. }\end{array}$} & \multirow[b]{2}{*}{ Total } \\
\hline & 1 & 2 & 3 & 4 & 5 & 6 & 7 & \\
\hline Hombre & $7.7 \%$ & $0.0 \%$ & $10.3 \%$ & $25.6 \%$ & $10.3 \%$ & $33.3 \%$ & $12.8 \%$ & $100.0 \%$ \\
\hline Mujer & $3.1 \%$ & $1.6 \%$ & $4.7 \%$ & $7.8 \%$ & $15.6 \%$ & $17.2 \%$ & $50.0 \%$ & $100.0 \%$ \\
\hline Total & $4.9 \%$ & $1.0 \%$ & $6.8 \%$ & $14.6 \%$ & $13.6 \%$ & $23.3 \%$ & $35.9 \%$ & $100.0 \%$ \\
\hline
\end{tabular}

${ }^{1}$ En una escala de $1=$ total desacuerdo a $7=$ total acuerdo

Fuente: Elaboración propia

La Tabla 4 nos indica que un $72.8 \%$ está de acuerdo con comprar productos hechos en México y apoyar el desarrollo del país. Un 14.6\% está indeciso, mientras que un $12.7 \%$ está en desacuerdo con la aseveración.

Tabla 5. Tabla de contingencia Género y Sólo aquellos productos que no estén disponibles en México deberían importarse. ${ }^{1}$

\begin{tabular}{|c|c|c|c|c|c|c|c|c|}
\hline \multirow[b]{2}{*}{ Sexo } & \multicolumn{7}{|c|}{$\begin{array}{l}\text { Solo aquellos productos que no estén disponibles en } \\
\text { México deberían importarse. }\end{array}$} & \multirow[b]{2}{*}{ Total } \\
\hline & 1 & 2 & 3 & 4 & 5 & 6 & 7 & \\
\hline \multirow[t]{2}{*}{ Hombre } & & & & & & & & \\
\hline & $10.3 \%$ & $2.6 \%$ & $15.4 \%$ & $15.4 \%$ & $12.8 \%$ & $15.4 \%$ & $28.2 \%$ & $100.0 \%$ \\
\hline \multirow[t]{2}{*}{ Mujer } & & & & & & & & \\
\hline & $1.6 \%$ & $3.1 \%$ & $10.9 \%$ & $28.1 \%$ & $21.9 \%$ & $10.9 \%$ & $23.4 \%$ & $100.0 \%$ \\
\hline \multirow[t]{2}{*}{ Total } & & & & & & & & \\
\hline & $4.9 \%$ & $2.9 \%$ & $12.6 \%$ & $23.3 \%$ & $18.4 \%$ & $12.6 \%$ & $25.2 \%$ & $100.0 \%$ \\
\hline
\end{tabular}

${ }^{1}$ En una escala de $1=$ total desacuerdo a $7=$ total acuerdo

Fuente: Elaboración propia

Con base en lo observado en la Tabla 5 un $56.2 \%$ está de acuerdo con importar únicamente aquellos productos que no están disponibles en México, un $23.3 \%$ está indeciso en su respuesta y un $20.4 \%$ está en desacuerdo con este ítem.

\section{Conclusión}

Los resultados del estudio nos muestran que, en general, la población entrevistada si se considera comprador etnocéntrico, pues evalúa en alto porcentaje las respuestas a las variables que así lo sugieren. Con base en los resultados obtenidos en esta investigación empírica con una muestra de 103 
sujetos validados, se confirma estadísticamente que no hay diferencias significativas entre hombres y mujeres con respecto a sus características etnocéntricas.

El alcance de este estudio es de nivel exploratorio y los resultados son preliminares debido a que el tamaño de la muestra no es representativo de la ciudad de Saltillo. Se pretenden como líneas futuras de investigación ampliar el número de participantes y llevar a cabo investigaciones comparativas con otros segmentos geográficos.

\section{References:}

1. Chung, C., Boyer, T., \& Han, S. (2009). Valuing quality attributes and country of origin in the Korean Market. Journal of Agricultural Economics, 682-698.

2. Hernández, S. R., Fernández, C. C., \& Baptista, L. P. (2014). Metodología de la Investigación (Sexta Edición). México: McGrawHill.

3. Muñoz-Penagos, C. A., \& Velandia-Morales, A. (Diciembre de 2014). Etnocentrismo del mercado colombiano y brasilero y su relación con las actitudes e intención de compra hacia productos extranjeros y domésticos. Suma Psicológica, 21(2), 99-106. doi:https://doi.org/10.1016/S0121-4381(14)70012-8

4. Schiffman, L. G., \& Lazar Kanuk, L. (2010). Comportamiento del Consumidor (DÉCIMA EDICIÓN ed.). México: PEARSON EDUCACIÓN.

5. Schnettler, B., Cid, E., Miranda, H., Sepúlveda, J., \& Denegri, M. (Julio de 2010). Etnocentrismo y Efecto País de Origen en la Compra de Arroz en Supermercados de las Ciudades de Los Angeles y Temuco, Chile. Panorama Socioeconómico, 28(40), 18-32.

6. Shankarmahesh, M. N. (2006). Consumer ethnocentrism: an integrative review of its antecedents and consequences international. Marketing Review(23), 146-172.

7. Shimp, T., \& Sharma, S. (1987). Consumer ethnocentrism: construction and validation of the CESTSCALE. Journal of Marketing Research, 24, 280-289.

8. Solomon, M. R. (2008). Comportamiento del consumidor. México: Pearson.

9. Sprotles, G. B., \& Kendall, E. L. (1986). A Methodology for Profiling Consumers' Decision-Making Styles. Journal of Consumer Affairs, 267-279. 